\title{
Water Cherenkov detector optimization for space weather studies in Antarctic
}

\author{
L. Otiniano $^{1 *}$, J. Peña ${ }^{2}$, J. Vega ${ }^{1}$, V.B. Valera ${ }^{1}$ and C. Castromonte ${ }^{1}$ for the LAGO \\ Collaboration $^{3}$, \\ ${ }^{1}$ Dirección de Astrofísica Comisión Nacional De Investigación y Desarrollo Aeroespacial \\ Lima, Perú \\ ${ }^{2}$ Universidad Industrial de Santander, Bucaramanga, Colombia \\ ${ }^{3}$ lagoproject.net/collab.html \\ E-mail: lotinianodconida.gob.pe
}

During the last two Peruvian summer campaigns in the Antarctica (2018 and 2019), two separate prototype water Cherenkov detectors of the LAGO project have been tested. As at high latitudes the lower geomagnetic shielding allows for the observation of cosmic rays lower than those at middle latitudes, the installation of a detector in the Antarctica is important for the development of the LAGO space weather program.

The objectives fulfilled in the second summer campaign were: a general improvement of the design of the detectors, wi-fi data transmission, autonomous operation, the elimination of light leaking and the optimization of the geometry for better separation of the electromagnetic / muonic components measured in the detector.

A comparison between the data obtained in the two Antarctic campaigns is shown, making emphasis in the measured flows and the strategy of using a second trigger level in the acquisition stage in order to perform a pre-processing of the data.

36th International Cosmic Ray Conference -ICRC2019-

July 24th - August 1st, 2019

Madison, WI, U.S.A.

\footnotetext{
* Speaker.
} 


\section{Introduction}

The Latinoamerican Giant Observatory (LAGO) is a network of Water Cherenkov Detectors (WCD) operated by a non-centralized collaborative network of Universities and Research Institutes in Latin America and Spain. This network is located in Latinoamérica at 7 diferent (operational) sites with altitudes, ranging from sea level (Lima, Perú) up to 5000 meters above sea level (Chacaltaya, Bolivia), and latitudes, which span most of Latin America from $18^{\circ} 59^{\prime} \mathrm{N}$ (Sierra Negra, Mexico) to $41^{\circ} 09^{\prime} \mathrm{S}$ (Bariloche, Argentina). The network covers an extensive range of geomagnetic shielding, and different levels of absorption and reaction at the atmosphere [1].
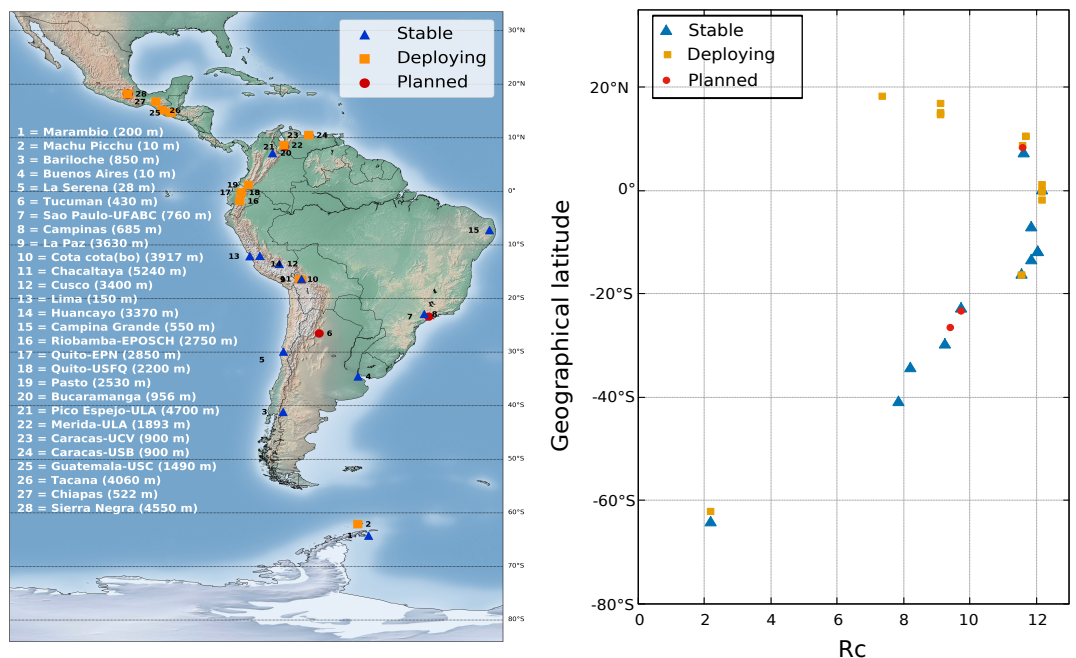

Figure 1: Geographical distribution and altitudes of the operational LAGO water Cherenkov detectors (bluetriangles), and those that are being deployed and will start its operation during the 2020-2021 biennium (redsquares). At the right panel, the vertical rigidity cut-off of each LAGO site is shown.

The Space Weather program of LAGO [4] is oriented to the study of the variations of the flow of secondary particles at ground level, that reflect the short and long term modulations of the galactic cosmic rays, providing information of the near space environment to Earth. Due to the geomagnetic shielding, particle detectors located at high latitudes allow the observation of cosmic rays (CRs) with lower energies than those located at middle or low latitudes. Thus, Antarctica is a privileged place to study CRs having the lowest energies that can be observed from ground level [3]. Actually LAGO project is development two sites: Argentine base Marambio [3] and Peruvian base Macchu Pichu. Here we describe the developments in detector optimization realized by the peruvian group and compare the test performed during the last two peruvian campaigns to Antartica (ANTAR XXV and ANTAR XXVI).

\section{Antarctica Deployment}

Peruvian campaign to Antarctic develops at summer season in January and February at the Macchu Picchu Scientific Station (ECAMP from spanish), it is located at Lat. $62^{\circ}$ 05' and Long. 

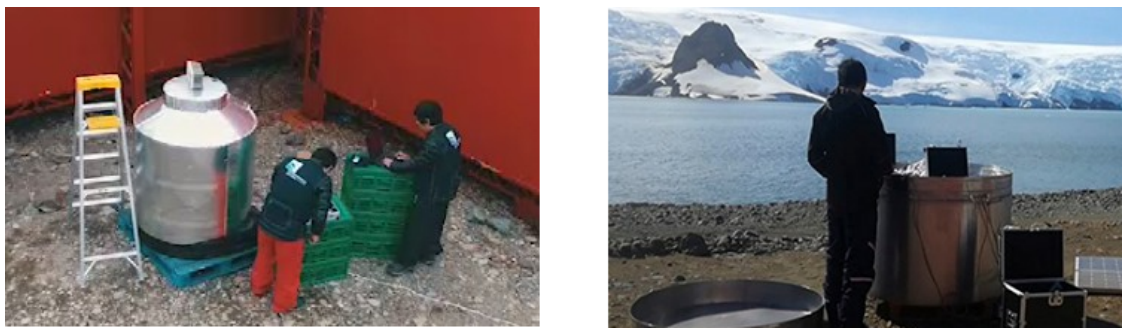

Figure 2: Deployment of WCD near peruvian Antartic station. Left: first detector, 2018. Rigth: second detector, 2019

$58^{\circ} 28^{\prime} \mathrm{W}$, at 15 meters above sea level. Principal problem at the station for deplyment is the lack of stable and continuous electric power and also the lack of space that the intense scientific activity demands in the short time of realization. During the XXV peruvian campaign (2018) we test our first WCD detector outside the station.

The first WCD of the LAGO site was manufactured with commercial cylindrical plastic tank containing a volume of 12001 ofpurified water, on top a large photomultiplier tube collects the Cherenkov radiation produced in the water by secondary CRs traversing the detector and reflected by an internal Tyvek coating. The detector is light isolated with different coatings (plastic an aluminium), see figure 2. The WCD signals are shaped and digitized by a custom made $40 \mathrm{MHz}$ electronic board controlled by a Digilent Nexys2 FPGA [8].

For the second peruvian Antarctic campaign (Antar XXVI, 2019), we develop a new custom detector optimized for muon/electromagnetic separation as we show in the next session. The assembly is far more easy than previous one. The detector in made from a custom stainless steal water tank with an internal cover of $\mathrm{TiO}_{2}$ based paint as substitute of Tyvek. Also the design uses and autonomous stable power supply. For protection of the operators from sudden changes in environmental conditions Wi-Fi communication with the station was necessary, see figure 2.

\section{Optimization of the WCD}

In order to calibrate the detector, we must estimate the expected charge distributions produced in the detector by Vertical Equivalent Muons (VEMs) [6]. In a previous work [5] we show a semianalitical characterization of the muon content measure in our first Antarctic detector. Based on [7] assuming a Gaussian distribution for the charge $(q)$ spectrum generated by VEMs $(V(q))$ that cross a cylindrical WCD, an analytical transformation of the charge spectrum $(F(q))$ generated by atmospheric muons is performed finding that:

$$
F(q)+\frac{q}{\alpha} F^{\prime}(q) \propto V(q)
$$

where $\alpha$ is a constant that depends on the track length distribution of muons in the detector.

Here we follow the opposite way, assuming that there is no change in $V(q)$ distribution, as first approximation, we calculate $F(q)$. In figure 3 we show this calculation, for the $V(q)$ estimation of the first detector calculated in [5]. 

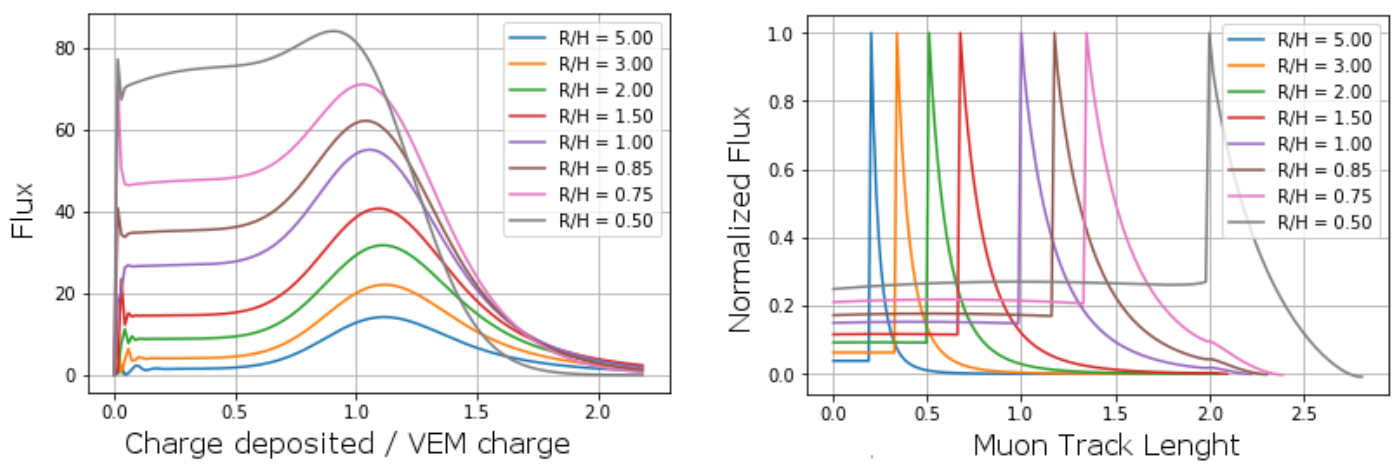

Figure 3: Left: expected charge distribution deposited by atmospheric muons in WCD. Right: expected track length of muon through the WCD for different of ratio R/H

The first $\mathrm{WCD}$ has a height $\mathrm{H}=100 \mathrm{~cm}$ and radius $\mathrm{R}=60 \mathrm{~cm}(\mathrm{R} / \mathrm{H}=0.6)$. For this configuration, from figure 3 , we expect that the muon charge distribution has a strong component of muons with a track lenght minor from vertical. To improve the muon peak we choose a ratio $\mathrm{R} / \mathrm{H}=1$ for our second $\mathrm{WCD}(\mathrm{H}=100 \mathrm{~cm}$ and radius $\mathrm{R}=60 \mathrm{~cm})$.

The signal produced by high energy particles going through the WCDs is a negative pulse with a sharp rise time of $\sim 10 \mathrm{~ns}$ and a decay time of $\sim 70$ ns produced by the attenuation length of Cherenkov photons in the detector. The LAGO acquisition system allows to digitize the pulses in a 10 bit resolution ADC ( $976 \mu \mathrm{V}$ per bin) at $40 \mathrm{MHz}$, packing the data in one hour files. The charge histogram of a WCD is obtained by time integration of the individual pulses measured in the WCD (in ADC units). As we expect that cosmic rays produce pulses with at least three bins of time we introduce a secondary trigger for the next bin (after trigger) at adquisition level with the same value of primary trigger (65 ADC), see figure 4 . This reduce the data files size from $200 \mathrm{Mb}$ per hour to $20 \mathrm{Mb}$. As internet data transmission is poor at ECAMP this is quite an improve for the operation.
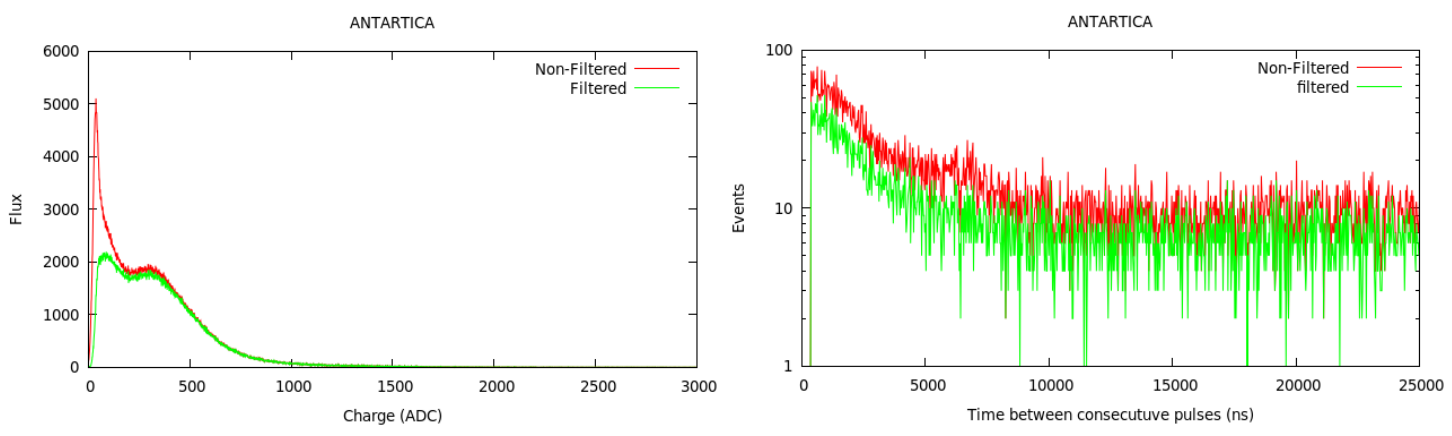

Figure 4: Right:Measured secondary cosmic ray charge spectrum (one hour) in the second detector with secondary trigger (green) and an with out (red)

In figure 5 we performed a muon selection (green) in the total charge spectrum (red) following the criteria used in [9]. Between two consecutive pulses, the one with the highest charge generated 
in the detector by secondary cosmic rays is chosen. The results from this selection are in good agreement with the estimations showed in figure 3 for both detectors.
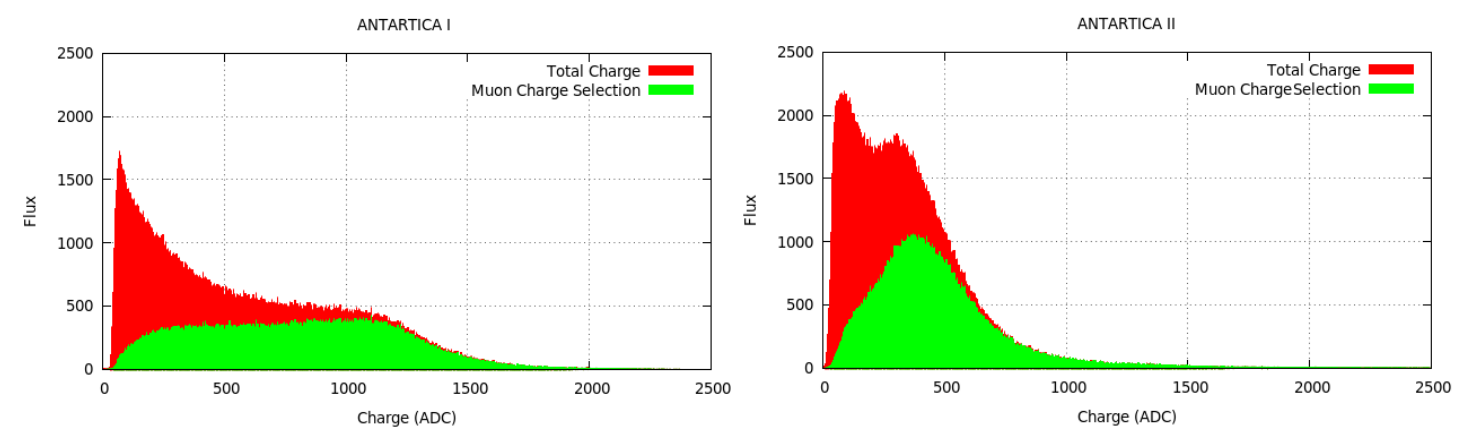

Figure 5: Measured secondary cosmic ray charge spectrum (one hour) and muon charge distribution estimation on Antarctic Peru's WCDs in red and muon selection applied (green)

LAGO calibration method looks for the second maximum in a charge histogram collected in one month and performs a Gaussian fit in the vicinity of this maximum, identifying this fitting function as the VEM distribution, where a central muonic band is defined. The limits in the band are given by the expected charge of muons that travel the maximum possible path in the detector (upper limit) and the transition point (local minimum) between the electromagnetic and the muonic regions of the spectrum. So it is very important that muon charge distribution to have a well resolved peak.

\section{Conclusion and Perspectives}

A method for fast evaluation of muon component in charge expectrum of WCD is under development. This method relies on a previous knowledge of the muon flux that traverse the detector and its geometry. But must been validated by simulations and independient measurements using secondary detectors ongoing. The method have been applied in the two detectors development for Antartica peruvian site of the LAGO network.

\section{Acknowledgments}

The autors gratefully acknowledges the financial support from Innovate-Perú: Fondo para la Innovación, Ciencia y Tecnología (FINCyT) trough the project PIBA-2-P-020-14 and the Ministerio de Relaciones Exteriores del Per ú through its Antarctic Affairs Direction. The LAGO Collaboration is very thankful to the Pierre Auger Collaboration for its continuous support.

\section{References}

[1] H. Asorey for the LAGO Collaboration. LAGO: the latin american giant observatory. Nuclear Instruments and Methods in Physics Research Section A: Accelerators, Spectrometers, Detectors and Associated Equipment, 2017. 
[2] M.S. Durán, H. Asorey, S. Dasso, L. Nuñez, Y. Peréz, C. Sarmiento for the LAGO Collaboration. The LAGO space weather program: Directional geomagnetic effects, background fluence calculations and multi-spectral data analysis, The 34th International Cosmic Ray Conference Vol. PoS (ICRC2015) (p. 142).

[3] Dasso, S., Masías-Meza, J., Gulisano, A. M., Asorey, H. A project to install water-cherenkov detectors in the antarctic peninsula as part of the LAGO detection network. The 34th International Cosmic Ray Conference, Vol. PoS (ICRC2015) (p. 105).

[4] M.S. Durán, H. Asorey, S. Dasso, L. Nuñez, Y. Peréz, C. Sarmiento for the LAGO Collaboration. The LAGO space weather program: Directional geomagnetic effects, background fluence calculations and multi-spectral data analysis, The 34th International Cosmic Ray Conference Vol. PoS (ICRC2015) (p. 142).

[5] Otiniano, L. and Sidelnik, I. for the Lago Collaboration. Development of a web monitor for the water Cherenkov detectors array of the LAGO project. Nuclear Instruments and Methods in Physics Research Section A: Accelerators, Spectrometers, Detectors and Associated Equipment 2019

[6] Bertou, X., et al. Calibration of the surface array of the Pierre Auger Observatory. Nuclear Instruments and Methods in Physics Research Section A: Accelerators, Spectrometers, Detectors and Associated Equipment 2006, 568, 2 P. 839-846

[7] Etchegoyen, A., et al. Muon-track studies in a water Cherenkov detector. Nuclear Instruments and Methods in Physics Research Section A: Accelerators, Spectrometers, Detectors and Associated Equipment 2005, vol. 545, no 3, p. 602-612.

[8] Haro, M. Sofo, L. H. Arnaldi, et al. The data acquisition system of the Latin American Giant Observatory (LAGO). Nuclear Instruments and Methods in Physics Research Section A: Accelerators, Spectrometers, Detectors and Associated Equipment 820 (2016): 34-39.

[9] Salazar, H., and L. Villasenor. Separation of cosmic-ray components in a single water Cherenkov detector. Nuclear Instruments and Methods in Physics Research Section A: Accelerators, Spectrometers, Detectors and Associated Equipment 553.1 (2005): 295-298. 